\title{
El abandono escolar temprano: ¿una cuestión de género? Comparando España y Nepal
}

\author{
Cabrera - Pérez, Lidia \\ Universidad de La Laguna, España \\ dcabrera@ull.edu.es \\ Dahal, Tanka \\ University of Dundee, UK \\ t.p.dahal@dundee.ac.uk
}

\section{Resumen}

El abandono escolar temprano es un asunto preocupante a nivel mundial. Todas las organizaciones internacionales como la OCDE, la Unión Europea, la UNESCO, incluyen entre sus objetivos y estrategias de desarrollo aumentar el periodo de edad de formación más allá de la edad obligatoria. Las diferencias de género en las tasas de abandono son muy destacadas y diferenciadas en todos los países. Mientras que en los países pobres son las niñas las que abandonan la escuela de forma muy temprana, en los países más desarrollados son los niños los que protagonizan porcentajes más elevados.

En este trabajo presentamos un análisis comparativo sobre abandono de la enseñanza obligatoria entre Nepal y España, con el objetivo de analizar cómo afecta el género en el abandono de estudios, y las implicaciones sociales que ello tiene para ambos grupos en los dos países. Para ello se compararon estadísticas oficiales y resultados obtenidos en dos estudios con 79 estudiantes de secundaria obligatoria (40 chicas y 29 chicos) en España, y 96 participantes (49 hombres y 47 mujeres, estudiantes, profesores y familiares) en Nepal.

Los resultados muestran diferencias importantes de género, si bien con tendencias estadísticas favorables hacia las chicas en España y muy desfavorables en Nepal, pero con consecuencias de desigualdad laboral y social para las mujeres en ambos países. En España las chicas tienen mejores notas y abandonan menos, sin embargo, tienen menos inserción laboral y ocupan menos cargos de alta cualificación. En Nepal, los porcentajes de abandono de las chicas ascienden hasta un $48,3 \%$ en educación primaria, a pesar del apoyo financiero recibido para que continúen estudiando. La cultura machista y la casta parece que tienen mucho peso sobre las iniciativas gubernamentales.

\section{Abstract}

The dropout studies is a complex educational problem worldwide. All international organizations such as the OECD, the European Union, UNESCO, include among their objectives and development strategies to increase the age of training beyond the compulsory school. The gender differences in dropout rates are very prominent and differentiated in all countries. While in poor countries it is girls who dropout of school very early, in the more developed countries it is boys who play the highest percentages.

In this paper, we write about a comparative analysis study of the dropout in compulsory education between Nepal and Spain. The aim was to analyze how the gender affects the dropout and the social implications that this has for both groups in the two countries. For this purpose, official statistics and results obtained in two studies are compared with 79 compulsory secondary students (40 girls and 29 boys) in Spain, and 96 participants (49 men and 47 women, students, teachers and relatives) in Nepal.

The results show important gender differences, although with favorable statistical trends towards girls in Spain and very unfavorable in Nepal, but with consequences of labor and social inequality for women in both countries. In Spain girls have better grades and drop less, however, they have less job insertion and occupy fewer highly qualified positions. In Nepal, the dropout rates for girls amount to $48.3 \%$ in primary school, despite the financial support received for them to continue studying. 'Gender' and in many parts of the country 'caste' are dimensions that are 'affecting literacy inequality' and consequently inequality in schooling outcome, against government initiatives.

Palabras clave: abandono de estudios, género, estudio comparativo.

Keywords: dropout studies, gender, comparative study. 


\section{INTRODUCCIÓN}

El abandono escolar temprano es un asunto preocupante a nivel mundial. Las cifras van disminuyendo, si bien de forma más lenta en algunas zonas del planeta que en otras. Todas las organizaciones internacionales como la OCDE, la Unión Europea, la UNESCO, incluyen entre sus objetivos y estrategias de desarrollo aumentar el periodo de edad de formación más allá de la edad obligatoria.

Sabates, Akyeampong, Wesbrook and Hunt (2010), en los países menos desarrollados y donde la escolarización no es obligatoria responsabilizan sobre todo a la pobreza. Ellos hablan de problemas de salud, malnutrición, desmotivación, obligaciones laborales, localización de las escuelas y de la calidad educativa que no ofrece los medios a los niños para su escolarización. También en los países más desarrollados son los ambientes familiares y sociales caracterizados por bajos recursos y poca formación los factores más relacionados (Marchena et al., 2014). Sin embargo, hay otras variables determinantes en la decisión de abandonar como el género. Las diferencias de género en las tasas de abandono son muy destacadas en todos los países. Mientras que en los países pobres son las niñas las que abandonan la escuela de forma muy temprana, en los países más desarrollados son los niños los que protagonizan porcentajes más elevados.

Sabates et al. (2010), a pesar de no encontrar tantas diferencias entre niñas y niños como entre ricos y pobres, muestran evidencias de la gran proporción de niñas entre 16 y 17 años que abandonan la escuela sin completar estudios primarios. El riesgo de que se queden embarazadas ha sido un factor apuntado por Colclough et al., 2000 y Leach et al., 2003, (Citado por Sabates et al., 2010). La casta a la que se pertenece (ethnicity-effect of caste), debido a las creencias sobre la educación de las niñas, es el factor más determinante en lagunas castas marginadas como Dalits, Madhesis y Janjatis. En general, el matrimonio precoz y el sistema familiar patriarcal es el aspecto sociocultural más influyente en el abandono escolar femenino en Nepal, pues las chicas al casarse tienen que hacerse cargo de la casa (Dahal,2018).

En los países más industrializados, por el contrario, las chicas se incorporan al mercado laboral mucho más tarde, y muchas no lo hacen nunca, por lo que dedican más tiempo a estudiar. Además, obtienen mejores calificaciones, repiten menos, logran más ventajas educativas (aprenden más), registran porcentajes de abandono 10 puntos por debajo de los chicos y las tasas de graduación son, para ellas, superiores en todos los niveles educativos. En las comunidades donde el nivel educativo de la población es más bajo, estas diferencias aumentan. El deseo claro de incorporación al mundo laboral de los chicos, sobre todo en chicos con bajos recursos económicos y muy desmotivados por los aprendizajes de la escuela, así como el afán de superación de las chicas en la lucha por la desigualdad, a la vez que la cultura social que hace a las chicas más obedientes y más disciplinadas a la hora de hacer las tareas escolares, son las variables más importantes que marcan la diferencia de rendimiento y abandono entre chicos y chicas.

Las ventajas educativas de las chicas, sin embargo, no se traducen en ventajas sociales, pues el acceso y promoción dentro del mundo laboral, contradictoriamente, es peor para ellas. Las chicas acceden en porcentajes inferiores a los chicos a la carrera investigadora, tienen más dificultades para encontrar empleo, trabajan en los peores lugares y promocionan menos que los hombres. Parece que las mujeres tienen que estudiar y prepararse más para trabajar en los mismos lugares que los hombres, o que los hombres no necesitan prepararse tanto para encontrar empleo debido a la oferta en sectores laborales tradicionalmente ocupados por hombres.

Galvez y Rodríguez (2011) han mostrado estas desigualdades laborales de género en las crisis económicas tanto en países pobres como ricos, evidenciando cómo cuando la economía es más solvente las mujeres son expulsadas del mundo laboral. Cuando las mujeres no trabajan tienen tres alternativas: formar una familia, cuidar a sus mayores o personas dependientes, o desarrollar una carrera profesional (estudiar, no incorporarse al mundo laboral), lo que explica el alto porcentaje de graduadas universitarias. En los países menos desarrollados, esta formación se realiza más en el seno familiar, al tratarse de una formación más dirigida al cuidado de la familia y al servicio doméstico.

En este contexto nos propusimos comparar cómo se dan esas diferencias de género en el abandono escolar temprano entre España y Nepal, con la finalidad de determinar el peso de la pobreza frente a otras variables culturales y de desigualdad entre hombre y mujeres. 


\section{CONTEXTO EDUCATIVO Y SOCIAL}

En España, la ampliación del periodo de enseñanza obligatoria a los 16 años (LOGSE, 1990), supuso un aumento de la población escolar, lo que favoreció el acceso a la educación de las clases sociales más desfavorecidas, a la vez que se incrementaron los porcentajes de suspenso y repetición, y como consecuencia el abandono de los estudios, la mayoría de las veces sin titulación. En 1994 el \% de abandono era de $36,4 \%$ y en 2004 de $31 \%$ (37\% chicos y $24,7 \%$ chicas). La variable más relacionada con el abandono siempre ha sido el suspenso y la repetición de curso (Mena, Fernández Enguita y Riviere, 2010). En la actualidad, los \% de repetición aumentan a medida que se avanza en el curso. Las tasas de idoneidad en los informes estadísticos de educación muestran que en $4^{\circ}$ grado de la ESO el 36,1\% del alumnado ha repetido curso alguna vez (MECD, 2017). En los últimos años se han puesto en práctica estrategias educativas, como los «Programas de diversificación curricular» sustituidos actualmente por los «Programas de Refuerzo», y los porcentajes se han reducido hasta 18,3\%. (2017). Las debilidades más importantes son: a) diferencias entre unas comunidades autónomas y otras ( País Vasco 7\% y Navarra 11,3\%; Baleares $26,5 \%$ y Andalucía 23,5\%) ;b) diferencias entre grupos nacionales y extranjeros (los niños con madres y padres extranjeros $(35,8 \%)$ abandonan más que los nacionales $(15,9 \%)$; c) diferencias entre niñas $(15 \%)$ y niños $(22,7 \%)$.

En Nepal, hasta la década de los 50 la educación casi no existía (Wood, 1959). La enseñanza se limitaba a la formación de sacerdotes y a la preservación de la cultura sánscrita (Sanskrit Pradhan Pathshala), que, por otro lado, estaba limitada a miembros de la Familia Real y los Ranas, gobernantes del país por más de 100 años. Cuando terminó el periodo de los Ranas, el \% de alfabetización del país era de un $5 \%$ (10\% hombres y $1 \%$ mujeres). Con el gobierno democrático de 1950 la educación pasó a ser un derecho para todos los ciudadanos nepalíes. El hecho más importante lo constituyó el National Education System Plan (NESP), introducido en 1973, y el Education for Rural Development Plan (ERDP), introducido en los años 80 (Basnet, 2013). Tras estas iniciativas se han obtenido importantes resultados hacia un desarrollo integral de la educación (Basnet, 2013). Sin embargo, la diversidad de grupos étnicos, basados en castas, ha marcado divisiones e importantes desigualdades que persisten en la actualidad, y que son desafíos educativos importantes. El sentimiento patriótico nacional del gobierno, unido a una política educativa guiada por objetivos de consolidación de ese sentimiento nacional y una política lingüística de enseñanza basada en una sola lengua, el «nepalés», ha caracterizado el curriculum educativo. La política lingüística única ha restado importancia y valor a la instrucción en la lengua materna, dificultando el acceso de los grupos minoritarios. El nacionalismo también se usó para reforzar y legitimar la posición dominante de los grupos de castas hindú de élite y el Shah monarchy (Caddell, 2007). La constitución posterior de 1990 defendió un «Nepal multiétnico, multilingüe, reino monárquico democrático, indivisible, soberano, hindú y constitucional» (Hutt 1993, citado por Caddell, 2007), sin embargo, se puso más que nunca de manifiesto la desigualdad entre unos grupos y otros, pues el sentimiento nacional del grupo dominante y los contenidos curriculares se desarrollaron de nuevo en torno a los del grupo elitista tradicionalmente educado. En los últimos años, las demandas de igualdad de oportunidades y calidad de la educación han sido, y continúan siendo, demandas crecientes, lo que ha llevado al gobierno a implantar el School Sector Reform Plan SSRP (2009-2015) para dar respuesta a las mismas. EI SSRP estructura el sistema educativo en dos niveles: Educación Primaria (de $1^{\circ}$ a $8^{\circ}$ ) y Educación Secundaria $\left(d e 9^{\circ}\right.$ a1 $2^{\circ}$ ).

Actualmente, la mayoría de las escuelas de educación secundaria son privadas, por lo que muchos niños y niñas en aldeas remotas no tienen acceso a la educación después del nivel primario, y más de la mitad de la población femenina se ve obligada a abandonar la escuela y casarse antes de los 18 años. Se han ido introduciendo algunas disposiciones legislativas para garantizar la paridad de género, a la vez que la casta, la raza o la religión. Estas medidas y los apoyos internacionales no han sido suficientes, pues las desigualdades de género aún persisten. Más del $70 \%$ de las mujeres no saben todavía leer ni escribir y más de 15,000 niñas son tratadas internacionalmente como esclavas sexuales. En el año 2015, la tasa general de alfabetización de adultos del país era del 63,9 por ciento $(76,4 \%$ hombres y $53,1 \%$ mujeres). Sin embargo, en algunas comunidades, como la comunidad rural del Himalaya, las diferencias de género son muy altas y el derecho a la educación no es el mismo para niños y niñas. Las niñas abandonan muy pronto la escuela, se comprometen, se casan y pasan el resto de su vida dependiendo de sus maridos, lo que las vuelve más vulnerables y convierte en objeto de violencia, abuso, inseguridad y tráfico sexual. Las razones que hacen que no todos los niños vayan a la escuela son las siguientes: son demasiado caras, están demasiado lejos, tienen que ayudar a sus familias, los padres no quieren que vayan a la escuela, los niños no quieren asistir (Thapa, 2013). 


\section{DIFERENCIAS DE GÉNERO}

La investigación sobre abandono y rendimiento centrada en el género es escasa. Sin embargo, se cuenta con muchas evidencias resultantes de estudios generales que muestran situaciones de desigualdad social, familiar y escolar, en ambos países, que explican las diferencias no sólo educativas sino también laborales y sociales.

Las dificultades de adaptación y desvinculación con la escuela por discrepancias entre la cultura social de procedencia y la cultura escolar han sido las variables más relacionadas. En España, estos estudiantes no están motivados con lo que estudian, repiten curso y pierden el interés por aprender y por la escuela. Repetir curso (Marchena y col. 2014) y llevar varios años suspendiendo asignaturas genera rechazo hacia al aprendizaje, y también afecta a la autoestima y autoconcepto, generando comportamientos poco ajustados (Rosales y Cabrera, 2017). Las niñas suelen tener más motivación intrínseca hacia el aprendizaje, encuentran placer en aprender y valoran la educación en sí misma no sólo como formación laboral (Cerezo y Casanova, 2004). En este contexto, las niñas tienen más éxito dado que encuentran en la educación una vía de superación personal y social (Martínez García, 2011), y estudian como un modo de protección a la futura discriminación laboral y social y como salida a la situación de desempleo. El nivel económico familiar también ha sido un factor constante, sin embargo, Marchena et al. (2014) en un estudio en Canarias encontraron que las razones económicas y laborales no eran los factores que estaban empujando a los jóvenes a dejar los estudios, sino el ambiente social y familiar. Rubio Castro (2009), explica que los chicos tienen más oportunidades en el marco laboral a pesar de sus bajos resultados escolares.

En Nepal, el acceso a las escuelas secundarias es limitado, sobre todo para las chicas, por la privatización de las mismas y por razones culturales de desigualdad que obliga a las niñas a abandonar la escuela a partir de la menarquía (aparición del primer periodo menstrual) para iniciarse en tareas de trabajo doméstico y de matrimonio. Por otro lado, cada grupo étnico trata de buscar su posición a través del reforzamiento de sus signos culturales (Pfaff-Czarnecka 1999), pero la atención a la diversidad cultural se ha aplicado sólo en la aceptación de los signos culturales de cada grupo, expresadas a través de sus ropas, comidas y costumbres, con lo que persisten las limitaciones para el acceso a los recursos de varios grupos sociales dentro de la población. Caddell (2007), explica los cambios y resultados educativos en Nepal a partir de lo que ella denomina «una naturaleza incrustada de las tensiones entre las visiones de la unidad nacional, modernidad y desarrollo», pero con una idea de desarrollo muy marcada por organizaciones e instituciones internacionales (Caddell, 2007). En este contexto, hay muchas dificultades para gestionar y atender la diversidad, en la que se ven afectados sobretodo grupos culturalmente minoritarios, discapacitados, mujeres y pobres. Las estadísticas muestran un \% mayor de niñas en escuelas públicas y un \% mayor de niños en escuelas privadas, lo que evidencia que las familias invierten más en la educación de los chicos (MOE,2016).

En relación a otras variables escolares y personales, en España se ha constatado una mejor adaptación de las niñas a la escuela, que son más sumisas, por lo que aceptan mejor las reglas de la escuela (Fernández-Enguita, 2009). Rubio Castro (2009), explica que dentro de la escuela el profesorado trata a niños y niñas de modo diferente, reforzando en ellas que sean ordenadas y obedientes y en ellos fuertes e inteligentes. Los modelos de conducta social en chicos y chicas han mostrado como los chicos huyen de parecer aplicados y buscan enfrentarse a la norma y a la disciplina escolar para conseguir liderazgo superar sus sentimientos de inferioridad. Las chicas son más maduras, no tienen necesidad de liderazgo, no sienten la presión del grupo de iguales como sus colegas masculinos y están siempre más dispuestas a colaborar (Rodríguez-Martínez y Blanco García,2015).

En Nepal, a pesar de que la primera razón es la pobreza, otra razón de abandono educativo es las malas notas o suspensos académicos (Thapa, 2013).

\section{UN ESTUDIO COMPARATIVO DE GÉNERO}

Nosotros hicimos un análisis comparativo con dos estudios. El primero se realizó en Tenerife (España) en el que participaron 69 estudiantes de secundaria obligatoria (40 chicas y 29 chicos). El segundo se realizó en Nepal con 96 participantes (49 hombres y 47 mujeres, estudiantes, profesorado y familiares). El objetivo de ambos estudios fue encontrar patrones explicativos a las diferencias de género en relación al abandono educativo temprano. 
En España, encontramos que, si bien no hay diferencias sociales y familiares entre chicos y chicas en políticas de igualdad educativa, y los encuestados informan que para sus padres y madres estudiar es muy importante, los chicos suspenden más (51\% había repetido curso frente al $31 \%$ de las chicas), lo que les predispone al abandono, y las chicas obtienen las mejores notas. Las chicas se adaptan mejor al centro, situándose 10 puntos por encima de los chicos cuando les pedimos que valoraran en una escala de 1 a 10 cómo les ha ido. Las chicas dedican muchas más horas al estudio diario fuera del colegio $(57,1 \%$ dedica más de una hora diaria de estudio) que los chicos $(27,6 \%$ dedica más de una hora diaria). Finalmente, las chicas $(2,4 \%)$ registran menos incidencias por mal comportamiento o indisciplina que los chicos $(11,5 \%)$.

Cuando se les preguntó por las variables que habían influido en sus notas, las chicas dicen que tienen buenas notas por la ayuda prestada por progenitores y profesorado y al esfuerzo personal, mientras que los chicos atribuyen sus bajos rendimientos a la influencia de los amigos. Las chicas sienten que suspenden porque no tienen capacidad para estudiar y los chicos sienten que suspenden porque los contenidos que estudian no les interesan y no se esforzaron lo suficiente. Finalmente, en relación a la percepción que tienen ambos grupos del estudio, para las chicas estudiar es muy importante, para comprender y saber más, para tener una buena imagen entre los demás y para satisfacer a sus padres. Para los chicos, estudiar es duro y aburrido y estudian para terminar las tareas cuanto antes, como una obligación.

En Nepal, las carencias en infraestructuras, recursos y ausencia de ambientes adecuados para las chicas, sistemas de seguridad y espacios físicos como baños para sus necesidades íntimas, llevó a que las estudiantes se sintieran excluidas de las escuelas públicas y abandonaran. Las políticas y objetivos educativos últimos también limitaron, según los informantes, el acceso a ciertos grupos étnicos y grupos geográficamente alejados, además de empujar más a las chicas a dejar la escuela por los suspensos en las pruebas finales (lo que denominan «presión sociopsicológica»). Cuando las chicas suspenden, sus padres pierden interés por su escolarización, y ellas tienen que soportar mucha vergüenza y presión psicológica y social por no ser capaces de llegar al nivel, lo que ha llevado a algunas niñas a suicidarse.

La división desigual del trabajo entre hombres y mujeres da preferencia a la educación de los niños sobre las niñas. Los niños encuestados fueron a escuelas consideradas de mejor calidad y las niñas a escuelas públicas. El liderazgo social de los miembros masculinos empuja a las familias a casar a sus hijas temprano, además del sistema de creencias y superstición de los padres sobre el sistema de dote y el efecto de la casta. Todo ello influye sobremanera en la deserción temprana de las niñas de la escuela.

\section{DISCUSIÓN Y CONCLUSIONES}

Los resultados en ambos estudios confirmaron nuestras hipótesis, además de coincidir con la literatura existente. A pesar de las grandes diferencias económicas, educativas y culturales, en ambos países se constataron diferencias de género en la educación en función del género, que redundan sobre todo en una mayor exigencia y menos oportunidades laborales para las chicas a pesar de la formación alcanzada.

Mientras en España la exigencia social, familiar y escolar consigue mejores resultados académicos y menor \% de abandono en las chicas, en Nepal esa exigencia ejerce para ellas una función de exclusión de la escuela y la educación. En ambos países, las mujeres siguen ocupando más responsabilidades en las tareas de maternidad y cuidados familiares, a la vez que son víctimas de maltrato y violencia. En Nepal la desigualdad y violencia es avalada por la cultura y legislación vigente que da el derecho a los hombres. En España, el poder de la formación femenina, que la sitúa muy por encima de los hombres, crea situaciones de desigualdad laboral al limitarle el acceso a estudios técnicos, al mundo laboral y al desarrollo profesional (debido a tareas de maternidad y cuidado de la familia). A la vez, a pesar de que no tenemos datos científicos que lo avalen, la desigualdad educativa a favor de la mujer creemos que está siendo un factor más de influencia en la situación de riesgo de las mujeres de ser víctimas de la cultura machista y de la violencia de género. 


\section{REFERENCIAS BIBLIOGRÁFICAS}

Basnet, L. D. (2013). Gender Discrimanation and Children's Right to Education In Nepal. Oslo: NTNU - Trondheim, Norwegian University of Science and Technology.

Caddell, Martha (2007). Education and change: A historical perspective on Schooling, Developing and the Nepali Nation-State. In: Kumar, Krishna and Oesterheld, Joachim eds. Education and Social Change. South Asia. New Delhi: Orient Longman, pp.251-284.

Cerezo, T. y Casanova, P. (2004). Diferencias de género en la motivación académica de los alumnos de Educación Secundaria Obligatoria. Revista Electrónica de Investigación Psicoeducativa, 2(1), 97-112.

Dahal, T. (2018). Impact of school system teaching and assessment on female students' dropout in Nepal (Unpublished PhD thesis). University of Dundee, Scotland, UK.

Fernández-Enguita, M. (2010). Fracaso escolar y abandono escolar en España. Barcelona: Obra Social La Caixa.

Gálvez Muñoz, L. y Rodríguez Madroño, P. (2011). La desigualdad de género en las crisis económicas. Investigaciones feministas, 2, 113-132.

Marchena, R., Martín, J.C., Santana, R. y Alemán, J. (2014). Riesgo de abandono escolar temprano y continuidad escolar en Canarias. Resumen ejecutivo. Gobierno de Canarias. Consejería de Educación, Universidades y Sostenibilidad. Documento consultado en: http://www.gobiernodecanarias.org/opencmsweb/export/sites/educacion/web/programas-redes-educativas/galerias/galeria_documentos/continuidadescolar/Resumen_Ejecutivo_Abandono_Escolar_Canarias.pdf.

Martínez García, J.S. (2011). Género y origen social: diferencias grandes en fracaso escolar administrativo y bajas en rendimiento educativo. Revista de la Asociación de la Sociología de la Educación, 4(3), 270-285.

Mena, L., Fernández-Enguita, M. y Riviére, J. (2010). Desenganchados de la educación. Procesos, experiencias, motivaciones y estrategias del abandono y el fracaso escolar. Revista de Educación, Número Extraordinario, 119-145.

Ministerio de Educación, Cultura y Deporte. Ley Orgánica de Ordenación General del Sistema Educativo LOGSE (1990). Disponible en: https://www.boe.es/boe/dias/1990/10/04/pdfs/A28927-28942.pdf

Ministerio de Educación, Cultura y Deporte. Secretaría General Técnica (2007). Datos y Cifras. Curso escolar $2017 / 18$. Consultado en Febrero de 2018 en: www.mecd.gob.es/servicios-al-ciudadano-mecd/dms/mecd/servicios-al-ciudadano-mecd/estadisticas/educacion/indicadores-publicaciones-sintesis/datos-cifras/Datosycifras1718esp.pdf.

MOE (2016). Education for All, National Review Report. Kathmandu: Ministry of Education.

Pfaff-Czarnecka, J. (1999). Debaring the State of the Nation: Ethnicization of Politics in Nepal - A Position Paper. In: J. Pfaff-Czarnecka / D.Rajasingham-Senanayake / A. Nandy / E.T. Gomez (eds.), Ethnic Futures: The State and Identity Politics in Asia. London: Sage, pp. 41-98.

Rodríguez-Martínez, C. y Blanco García, N. (2015). Diferencias de género, abandono escolar y continuidad en los estudios. Revista Iberoamericana de Educación, 68, 59-78.

Rosales González, C. y Cabrera Pérez, L. (2017). Causas y soluciones de los bajos resultados educativos de Canarias mostrados en los informes de evaluación. QURRICULUM. Revista de Teoría, Investigación y Práctica Educativa, $N^{\circ} 30$, 157-181. Disponible en: http://publica.webs.ull.es/publicaciones/detalle/revista-qurriculum/qurriculum-revista-de-teoria-investigacion-y-practica-educativa/.

Rubio Castro, P. (2009). El abandono y falta de éxito de los chicos en la escuela, un problema de género. CEE Participación educativa, 11, 23-29. Consultado en: file: //Users/lidiacabrera/Downloads/Monogr\%C3\%A1fico_El\%20abandono\%20y\%20la\%20falta\%20de\%20\%C3\%A9xito\%20de\%20los\%20chicos\%20en\%20la\%20escuela\%20(1).pdf.

Sabates, R., Akyeampong, K., Wesbrook, J. and Hunt, F. (2010). School Drop out: Patterns, Causes, Changes and Policies. Paper commissiones for the EFA Global Monitoring Report 2011, The hidden crisis: Armed conflict and education. Disponible en:http://unesdoc.unesco.org/images/0019/001907/190771e.pdf.

Tapha, Surya B. (2013). Relationship between education and poverty in Nepal. Economic Journal of Development Issues, 15(16), 148-161.

Wood, H. B. (1959). Development of Education in Nepal. Educational Leadership, 429-433. 